\title{
Health Care Resource Utilization and Costs for Patients with Tardive Dyskinesia
}

\author{
Benjamin Carroll, PharmD, and Debra E. Irwin, PhD, MSPH
}

\begin{abstract}
BACKGROUND: Tardive dyskinesia (TD) is an often-irreversible movement disorder affecting any part of the body. Patients experience debilitating symptoms that lower quality of life and increase mortality. Prolonged exposure to dopamine antagonists, which are frequently prescribed for psychiatric disorders as neuroleptic (antipsychotic) drugs, is a common cause of TD. The estimated prevalence of TD is $20 \%-50 \%$ among patients on antipsychotics, and the reported incidence of TD ranges from $<1 \%$ to $42 \%$, depending on the antipsychotics studied. However, there are few real-world studies examining health care utilization and the economic burden of TD.

OBJECTIVE: To assess health care utilization and costs in a sample of patients with TD from the commercially insured and Medicare supplemental U.S. populations.

METHODS: A retrospective analysis was conducted using Truven MarketScan Commercial and Medicare administrative claims data. Patients were included in the TD group if they had the first TD diagnosis (index date) between January 1, 2008, and September 30, 2014, with $\geq 1$ inpatient or $\geq 2$ outpatient nondiagnostic claims for TD (ICD-9-CM code 333.85). Patients without TD were randomly assigned an index date. Further inclusion criteria for all patients were $\geq 12$ months of pre- and post-index medical and pharmacy continuous enrollment and no evidence of TD claims during the pre-index period. Patients with TD were directly matched to patients without TD within subgroups for schizophrenia, major depressive disorder, bipolar disorder, and other psychiatric disorders and propensity matched on other demographic and clinical factors. Descriptive statistics on the incidence of resource utilization and costs of health care were reported.
\end{abstract}

RESULTS: Of 3,397 patients with TD, 834 met the selection criteria and were matched to 834 non-TD controls. Patients with TD generally had significantly greater utilization during the 12 months after TD diagnosis than in the 12 months before TD diagnosis. Their rates for health care utilization and costs were also substantially higher than for those without TD. During the post-TD-diagnosis time, inpatient admissions ( $55.5 \%$ vs. $26.1 \% ; P<0.001)$ and emergency room visits $(61.5 \%$ vs. $50.6 \% ; P<0.001)$ occurred more for patients with TD than for patients without TD. Total health care costs were significantly greater for patients with TD than for those without TD ( $\$ 54,656$ vs. $\$ 28,777 ; P<0.001)$.

CONCLUSIONS: Patients diagnosed with TD demonstrate significantly higher health care utilization and costs compared with non-TD patients.

J Manag Care Spec Pharm. 2019;25(7):810-16

Copyright $\odot 2019$, Academy of Managed Care Pharmacy. All rights reserved.

\section{What is already known about this subject}

Tardive dyskinesia (TD) has an estimated prevalence of $25.3 \%$ among patients who receive antipsychotics.

The prevalence of TD is anticipated to rise because of increasing antipsychotic use, including expanded off-label use for conditions such as anxiety, post-traumatic stress disorder, obsessive compulsive disorder, and eating disorders.

Most published studies on the economic burden of antipsychotics focus on clinical trial data and estimate costs related to specific treatments for psychiatric disorders or to the overall disease burden of illness (e.g., costs associated with schizophrenia rather than TD-specific costs).

\section{What this study adds}

This study used real-world data to demonstrate an association between TD diagnosis and substantially more health care resource utilization; this increased utilization resulted in a $26 \%$ increase in total health care costs.

Compared with those without TD, patients with TD have $29 \%$ more inpatient admissions, 34\% of which are TD-specific; $5 \%$ more outpatient office visits, $54 \%$ of which are TD-specific; and $21 \%$ more emergency room visits.

$\mathrm{T}$ ardive dyskinesia (TD) is a hyperkinetic movement disorder characterized by repetitive, purposeless, and involuntary movements in the oro-buccal-lingual region or of the extremities. ${ }^{1,2}$ TD is typically caused by medications that block dopamine receptors, which are commonly prescribed as neuroleptic (antipsychotic) drugs to treat psychiatric disorders. $^{3}$ With a resolution rate of only 13\%, TD is often irreversible and has been linked to a poor quality of life with increased medical morbidity and mortality. ${ }^{4-8}$

Strategies to suppress TD include careful reduction of the causative agent or switching to a different antipsychotic drug. However, lowering the dose of or discontinuing antipsychotics increases the risk of relapse; furthermore, it can exacerbate TD, with an even lower chance of reversibility. ${ }^{9,10}$ Before April 2017, there were no medications for the treatment of TD approved by the U.S. Food and Drug Administration (FDA). The only off-label medication was tetrabenazine, a reversible vesicular monoamine transporter 2 (VMAT2) inhibitor approved for Huntington disease chorea. ${ }^{11}$ However, tetrabenazine has 
been associated with adverse effects, including depression and suicidality. ${ }^{12}$ Recently, 2 novel VMAT2 inhibitors, valbenazine and deutetrabenazine, showed improved safety profiles and received FDA approval for the treatment of TD. Currently, affordability may be the major obstacle for patients to receive treatment with either valbenazine or deutetrabenazine. ${ }^{13}$

In 2017, the global mean prevalence of TD among all patients taking antipsychotics was estimated to be $25.3 \%$, with great variation among the different types of antipsychotics and other clinical parameters, such as age group. ${ }^{14}$ The reported incidence of TD in adults receiving newer or older antipsychotic drugs ranges from $<1 \%$ to $42 \%$ per year. ${ }^{1,15}$ Elderly patients are up to 5 times more likely to get TD than younger patients treated with conventional antipsychotics. ${ }^{16}$ Other risk factors, including the type of underlying mental illness, longterm use and higher doses of antipsychotics, and pre-existence of other movement disorders, may also have a substantial effect on the incidence of TD. ${ }^{17}$ Owing to the continuous rise in the overall number of antipsychotic-treated patients in the United States, the prevalence of TD is likely to increase steadily. ${ }^{18,19}$

To date, there is a lack of literature on the economic burden of TD on patients receiving antipsychotics, despite its widespread prevalence. Most published studies focus on clinical trial data or do not specifically focus on TD costs; instead, they estimate costs related to specific treatments for psychiatric disorders or to the overall disease burden of illness (e.g., costs of schizophrenia or comparing costs related to 2 different antipsychotic medications). ${ }^{20,21}$ The objective of this study was to add to the existing literature by describing health care utilization and costs in a sample of patients with TD from the commercially insured and Medicare supplemental U.S. populations.

\section{Methods}

\section{Data Sources}

A retrospective cohort analysis was conducted using administrative medical and pharmacy claims data from the Truven MarketScan Commercial Claims and Medicare administrative claims databases for the period between January 1, 2007, and September 30, 2015.

The MarketScan Commercial Claims database contains complete longitudinal records of the inpatient, outpatient, and outpatient prescription drug experience of approximately 132.0 million employees and their dependents, covered under a variety of fee-for-service and managed care health plans across all U.S. geographic regions.

The MarketScan Medicare Supplemental and Coordination of Benefits database contains the health care experience (medical and pharmacy) of approximately 9.9 million retirees with Medicare supplemental insurance.

All databases were deidentified in compliance with the Health Insurance Portability and Accountability Act (HIPAA).

\section{Study Population}

Patients in the TD cohort were diagnosed with TD for the first time (index date) between January 1, 2008, and September 30, 2014 , with $\geq 1$ inpatient or $\geq 2$ outpatient nondiagnostic claims for TD (International Classification of Diseases, Ninth Revision, Clinical Modification [ICD-9-CM] code 333.85) on different days, but no more than 365 days apart. All patients were aged $\geq 18$ years on the index date; had continuous medical and pharmacy enrollment for $\geq 12$ months before and after the index date; and had no evidence of nondiagnostic TD claims during the 12 months before the index date.

Patients who were considered potential controls in the nonTD cohort had $\geq 1$ inpatient or $\geq 2$ outpatient nondiagnostic claims on different days, but no more than 365 days apart, for schizophrenia, bipolar disorder, major depressive disorder, or other psychiatric disorders (ICD-9-CM codes in the Appendix, available in online article) or had pharmacy claims for atypical or typical antipsychotics between January 1, 2008, and September 30, 2014. The potential non-TD controls were not necessarily newly diagnosed for their mental health diagnosis. All of these patients were aged $\geq 18$ years on the index date, which was randomly assigned based on the index date distribution in the TD cohort; had continuous medical and pharmacy enrollment for $\geq 12$ months before and after the index date; and had no evidence of nondiagnostic TD claims during the study period.

\section{Propensity Score Matching}

The matching of patients with TD to control patients without TD was implemented to reduce the bias that could potentially exist in an observational claims data study. TD patients without an underlying psychiatric disorder or antipsychotic drug use were not included in the match, leaving 834 TD patients available for the matched analysis. In order to obtain balance between the 2 cohorts for other covariates, a combination of direct matching and propensity score matching was used.

Several mutually exclusive strata were created for both cohorts (TD cases and potential non-TD controls) based on underlying disease status: schizophrenia only; bipolar disorder only; major depressive disorder only; schizophrenia and bipolar disorder; schizophrenia and major depressive disorder; bipolar disorder and major depressive disorder; all 3 disorders (schizophrenia, bipolar, and major depressive disorder); and no evidence of schizophrenia, bipolar, or major depressive disorder but with diagnoses of other psychiatric disorders. Five potential control patients were randomly selected within each disease stratum, allowing for the 2 cohorts to be directly matched on underlying disease status.

Next, propensity score matching was performed using demographic variables (age, gender, payer); pre-index clinical characteristics (Deyo Charlson Comorbidity Index score, comorbid diagnosis of anxiety, drug/alcohol dependence, 


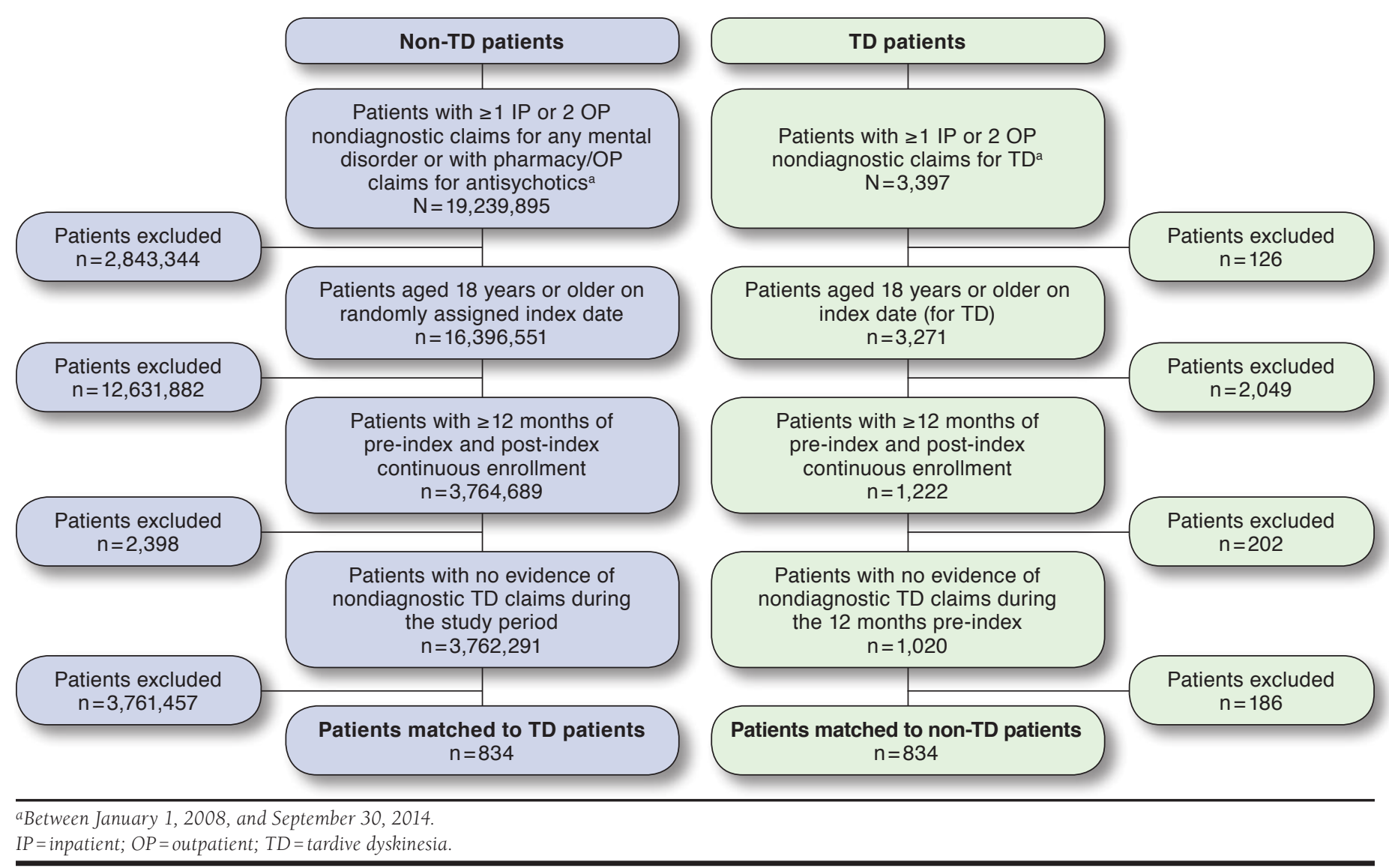

dementia, and other mental health conditions); and medication use including antipsychotics (atypical and typical), antidepressants, anxiolytics and other medications known to be associated with TD (e.g, anticholinergics, promethazine, and metoclopramide); and health care utilization patterns (pre-index all-cause health care cost and mental health-related hospitalizations and emergency department visits). The quality of the match was evaluated using a standardized difference for each matching covariate, and each patient group was considered balanced with respect to a given covariate if its corresponding standardized difference was less than 10\%. The final matched cohorts included 834 patients with TD matched to 834 patients without TD.

\section{Outcomes}

Demographics were measured on the index date, and clinical characteristics, comorbid conditions, and concomitant medications were assessed during the pre-index period.

All-cause health care utilization (inpatient admissions, lengths of stay, admissions per patient, emergency room visits, and outpatient office visits) and costs (inpatient, outpatient, and pharmaceutical) were measured during the 12-month preindex and post-index periods in the matched cohorts. Among patients with TD, TD-specific health care utilization and costs were evaluated based on claims with the diagnosis code for TD (ICD-9-CM code 333.85) in any position, and TD-specific pharmacy costs (sum of costs for tetrabenazine, clonazepam, and amantadine) were also included. Average health care costs and resource utilization were calculated among all patients, serving as the denominator. Health care costs were measured based on paid amounts of adjudicated claims, including insurer and health plan payments, as well as patient cost sharing in the form of copayment, deductible, and coinsurance. Cost for services provided under capitated arrangements were estimated using payment proxies that were computed based on paid claims at the procedure level using the MarketScan Commercial and Medicare Supplemental databases. All dollar estimates were inflated to 2015 dollars using the medical care component of the Consumer Price Index. 


\section{TABLE 1}

\begin{tabular}{|c|c|c|c|}
\hline \multirow[b]{2}{*}{ Age, years (SD) } & \begin{tabular}{|c|} 
Non-TD Patients \\
Analyzed \\
$(\mathrm{n}=834)$
\end{tabular} & \multicolumn{2}{|c|}{$\begin{array}{l}\text { TD Patients } \\
\text { Analyzed } \\
(\mathbf{n}=834)\end{array}$} \\
\hline & $63.5(16.7)$ & 63.4 & $(15.1)$ \\
\hline \multicolumn{4}{|l|}{ Sex, \% (n) } \\
\hline Male & $30.3 \quad(253)$ & 30.3 & $(253)$ \\
\hline Female & $69.7 \quad(581)$ & 69.7 & $(581)$ \\
\hline \multicolumn{4}{|l|}{ Database, \% (n) } \\
\hline Commercial & $46.5 \quad(388)$ & 50.1 & $(418)$ \\
\hline Medicare & $53.5 \quad(446)$ & 49.9 & $(416)$ \\
\hline \multicolumn{4}{|l|}{ Urbanicity, \% (n) } \\
\hline Urban & $85.9 \quad(716)$ & 84.3 & $(703)$ \\
\hline Rural & $13.2(110)$ & 15.2 & $(127)$ \\
\hline Unknown & $(8)$ & 0.5 & (4) \\
\hline \multicolumn{4}{|c|}{ Underlying disease status used in matching, a \% (n) } \\
\hline Bipolar disorder & $33.8 \quad(282)$ & 33.8 & $(282)$ \\
\hline Major depressive disorder & $36.6 \quad(305)$ & 36.6 & $(305)$ \\
\hline Schizophrenia & $18.3(153)$ & 18.3 & (153) \\
\hline Other psychiatric disorders ${ }^{b}$ & \begin{tabular}{|l|l|}
$37.6(314)$ \\
\end{tabular} & 37.6 & $(314)$ \\
\hline \multicolumn{4}{|c|}{ Selected common ${ }^{\mathrm{c}}$ comorbid conditions during the pre-index period, \% (n) } \\
\hline Anxiety & $31.4(262)$ & 31.9 & $(266)$ \\
\hline Dementia & $14.2 \quad(118)$ & 15.4 & $(128)$ \\
\hline Diabetes & $22.3 \quad(186)$ & 27.2 & $(227)^{\mathrm{e}}$ \\
\hline Extrapyramidal symptoms $^{\mathrm{d}}$ & $3.6 \quad(30)$ & 18.5 & $(154)^{\mathrm{e}}$ \\
\hline Hyperlipidemia & $37.4 \quad(312)$ & 38.5 & $(321)$ \\
\hline Hypertension & \begin{tabular}{|l|l|}
$55.6(464)$ \\
\end{tabular} & 54.9 & $(458)$ \\
\hline \multicolumn{4}{|c|}{ Selected concomitant medications ${ }^{c}$ during the pre-index period, \% (n) } \\
\hline Atypical antipsychotics & $52.8(440)$ & 50.6 & $(422)$ \\
\hline Typical antipsychotics & $15.5 \quad(129)$ & 17.9 & $(149)$ \\
\hline Antidepressants & $68.2(569)$ & 69.8 & $(582)$ \\
\hline Anticholinergics & $24.9 \quad(208)$ & 29.4 & $(245)^{\mathrm{e}}$ \\
\hline Anxiolytics & $28.7 \quad(239)$ & 29.6 & $(247)$ \\
\hline Clonazepam & $23.4 \quad(195)$ & 22.2 & $(185)$ \\
\hline Promethazine & $16.2 \quad(135)$ & 12.7 & $(106)^{\mathrm{e}}$ \\
\hline \multicolumn{4}{|c|}{$\begin{array}{l}\text { aPatients may have more than } 1 \text { condition. } \\
\text { b Other psychiatric diagnoses coded in ICD-9-CM code range of 290.xx-319.xx, not } \\
\text { including schizophrenia, bipolar, or major depressive disorder. } \\
\text { cPresent in }>15 \% \text { of patients. } \\
\text { dIncludes ICD-9-CM codes 332.1, 333.0-333.3, 333.5-333.7, 333.81, 333.82, } \\
333.83,333.89 \text {, and 333.9. } \\
\text { eP<0.05 versus non-TD patients. } \\
\text { ICD-9-CM = International Classification of Diseases, Ninth Revision, Clinical } \\
\text { Modification; SD = standard deviation; TD = tardive dyskinesia. }\end{array}$} \\
\hline
\end{tabular}

\section{Statistical Analysis}

Univariate and bivariate (where appropriate) descriptive analyses, including demographic and clinical characteristics for the overall sample, were summarized. Statistical tests of significance were performed for differences between the pre- and post-index periods. Chi-square tests were used to evaluate the statistical significance of differences for categorical variables (presented as counts and percentages). For continuous variables (presented as means and standard deviation), t-tests or analysis of variance were used with $P<0.05$ set a priori as the threshold for statistical significance.

\section{Results}

\section{Study Population Characteristics Among Matched TD and Non-TD Cohorts}

Among 3,397 patients identified as having $\geq 1$ inpatient or 2 outpatient nondiagnostic claims for TD between January 1 , 2008, and September 30, 2014, 834 patients met the selection criteria and were matched to 834 non-TD-controls (Figure 1). The demographic and clinical characteristics, comorbid conditions, and concomitant medications of the study population are summarized in Table 1. After matching, most demographic and clinical characteristics were similar between the TD and non-TD cohorts. Both cohorts had a mean age of approximately 63 years. Approximately $85 \%$ of patients resided in urban areas, and just over two thirds $(\sim 70 \%)$ of patients were female. Approximately half of the patients had commercial insurance, and the other half had Medicare.

The most common comorbid conditions during the preindex period were hypertension (TD: 54.9\%, non-TD: 55.6\%, $P>0.05$ ); hyperlipidemia (TD: 38.5\%, non-TD: 37.4\%, P>0.05); and anxiety (TD: $31.9 \%$, non-TD: $31.4 \%, P>0.05$ ). Diabetes was more common among patients with TD than without TD (TD: $27.2 \%$, non-TD: $22.3 \%, P<0.05$ ). The most common concomitant medications were antidepressants (TD: 69.8\%, non-TD: $68.2 \%, P>0.05$ ); atypical antipsychotics (TD: 50.6\%, non-TD: $52.8 \%, P>0.05$ ); and anxiolytics (TD: $29.6 \%$, non-TD: $28.7 \%, P>0.05)$. The matched TD cohort and the non-TD cohort had similar proportions of patients using atypical antipsychotics (TD: 50.6\%, non-TD 52.8\%, P>0.05), but significantly higher proportions of TD patients than non-TD patients used some of the specific atypical antipsychotics (paliperidone TD: 2.0\%, non-TD: $0.7 \%, P=0.0209$; risperidone TD: $14.6 \%$, non-TD: 10.9\%, $P=0.0230$; and ziprasidone TD: $7.3 \%$, non-TD: $3.8 \%$, $P=0.0020$ ). In addition, there were some other significantly higher rates of use among TD patients than in non-TD patients for other medications used, such as anticholinergics (TD: 29.4\%, non-TD: $24.9 \%, P=0.0417$ ); metoclopramide (TD: 9.6\%, nonTD: $6.6 \%, P=0.0248$ ); and amantadine (TD: $5.0 \%$, non-TD: $0.5 \%, P<0.0001$ ), with lower use of promethazine in the TD versus non-TD cohort (TD: $12.7 \%$, non-TD: $16.2 \%, P=0.0434$ ).

\section{Health Care Resource Utilization}

In the TD group, assessment of health care utilization showed significantly more inpatient admissions (pre-index: 40.0\%, post-index: $55.5 \%, P<0.001$; Figure 2); more admissions per patient (pre-index: 0.8, post-index: 1.1, $P<0.001$ ); and longer lengths of stay (pre-index: 3.3 days, post-index: 4.8 days, $P<0.001)$ in the post-index period than in the pre-index period. Additionally, overall emergency room visits were significantly higher (pre-index: 54.4\%, post-index: $61.5 \%, P=0.003$; Figure 2) after diagnosis than during the prediagnosis period, whereas the number of emergency room visits per patient remained similar (pre-index: 1.6 , post-index: $1.9, P>0.05$ ). The overall percentage of patients with outpatient visits was high before and 

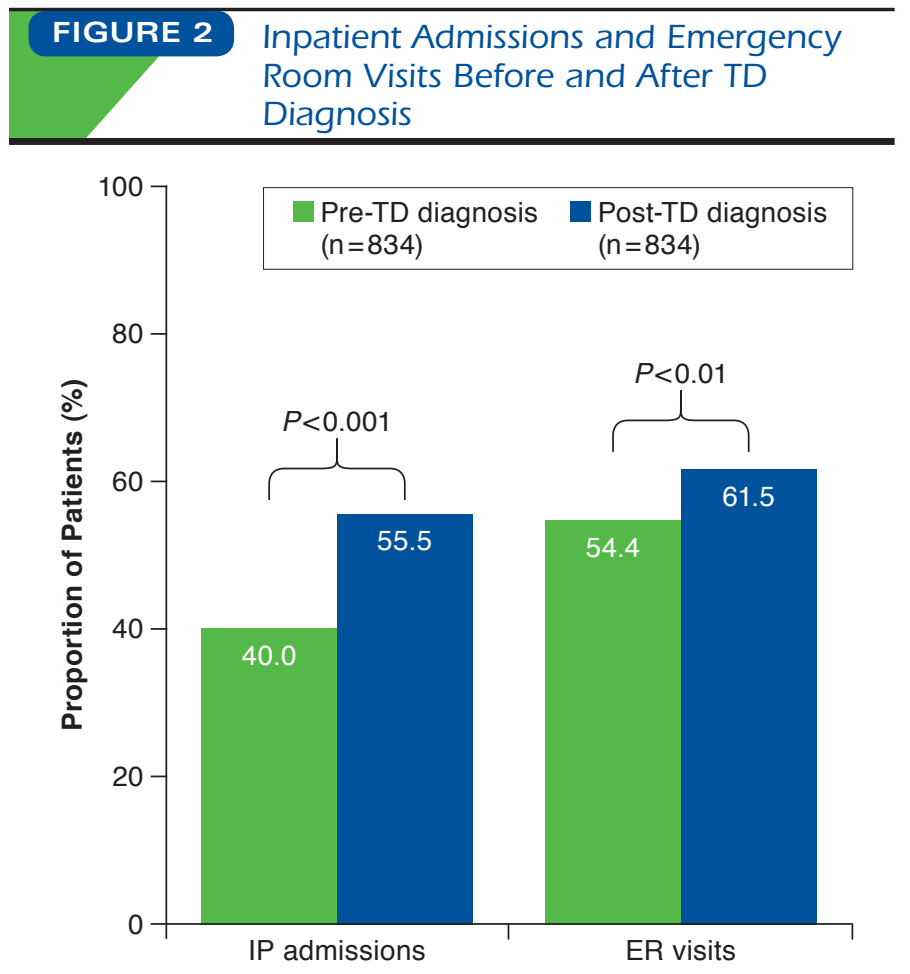

Note: Percentage of patients with any IP admission and percentage of patients with any ER visit before versus after TD diagnosis.

$E R=$ emergency room; $I P=$ inpatient $; T D=$ tardive dyskinesia

after diagnosis, with $96.0 \%$ and $95.4 \%$ of patients experiencing a visit during pre- and post-index periods, respectively. The number of outpatient visits per patient, however, was greater after diagnosis (pre-index: 13.1, post-index: 15.7, $P<0.001$ ).

Among the 2 matched cohorts, pre-index all-cause health care resource utilization was similar (inpatient admissions TD: $40.0 \%$, non-TD: $38.7 \%, P>0.05$; average length of stay TD: 3.3, non-TD: $2.9, P>0.05$; emergency room visits TD: 54.4\%, non-TD: 51.2\%, $P>0.05$; and outpatient visits TD: 96.0\%, non-TD: $94.2 \%, P>0.05)$. During the post-index period, patients with TD had higher rates of all-cause inpatient admissions (TD: 55.5\%, non-TD: 26.1\%, $P<0.001$; Figure 3); more admissions per patient (TD: 1.1, non-TD: 0.4, $P<0.001$ ); and longer average lengths of stay (TD: 4.8 days, non-TD: 1.8 days, $P<0.001)$ compared with patients without TD. Among all patients with TD ( $\mathrm{n}=834), 34.2 \%$ of inpatient admissions were specific for TD. Additionally, for patients with TD overall emergency room visits (TD: 61.5\%, non-TD: 40.6\%, $P<0.001$; Figure 3) and emergency room visits per patient (TD: 1.9, non-TD: 1.0, $P<0.001$ ) were significantly higher than for those without TD. Among patients with TD, the proportion of TD-related emergency room visits was 8.6\%.

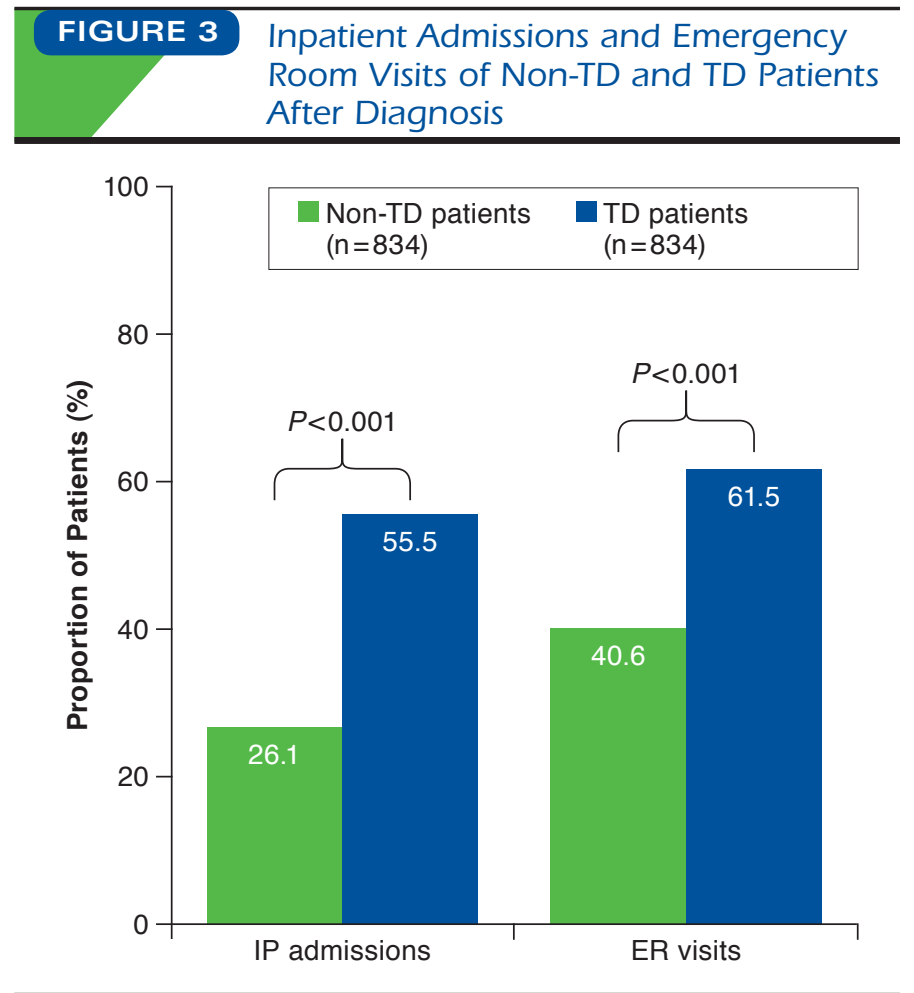

Note: Percentage of patients with any ER visit pre-index versus post-index. Mean ER visits for individual patients, pre-index versus post-index.

$E R=$ emergency room; IP = inpatient; $T D=$ tardive dyskinesia .

The overall percentage of outpatient office visits (TD: 95.4\%, non-TD: $90.6 \%, P<0.01$, with $54.2 \%$ being TD-specific) and the number of outpatient office visits per patient (TD: 15.7, non-TD: 10.4, $P<0.001$ ) were higher in the TD cohort compared with the non-TD cohort during the post-index period.

\section{Health Care Costs}

The all-cause healthcare costs were similar between the 2 cohorts before the index date (TD: $\$ 43,312$, non-TD: $\$ 40,202$, $P>0.05)$. For patients with TD, the mean total all-cause health care costs increased by $26.2 \%(P=0.003)$, from $\$ 43,312$ in the pre-index period to $\$ 54,656$ in the post-index period. The major cost driver was inpatient admissions (TD: $\$ 23,486$, non-TD: $\$ 15,049, P=0.001$ ), with an increase of $56.1 \%$ in the post-index period compared with the pre-index period. After the index date, costs for inpatient admissions (TD: $\$ 23,486$, non-TD: $\$ 9,053, P<0.001$ ); outpatient services (TD: $\$ 22,930$, non-TD: $\$ 13,186, P<0.001$ ); and outpatient pharmacy (TD: $\$ 8,241$, non-TD: $\$ 6,538, P=0.002$ ) were substantially higher for the TD cohort than for the matched non-TD cohort (Figure 4), thus, leading to significantly higher total all-cause health care costs for patients with TD (TD: $\$ 54,656$, non-TD: $\$ 28,777$, $P<0.001)$. Patients with TD spent an average of $\$ 5,408$ on 


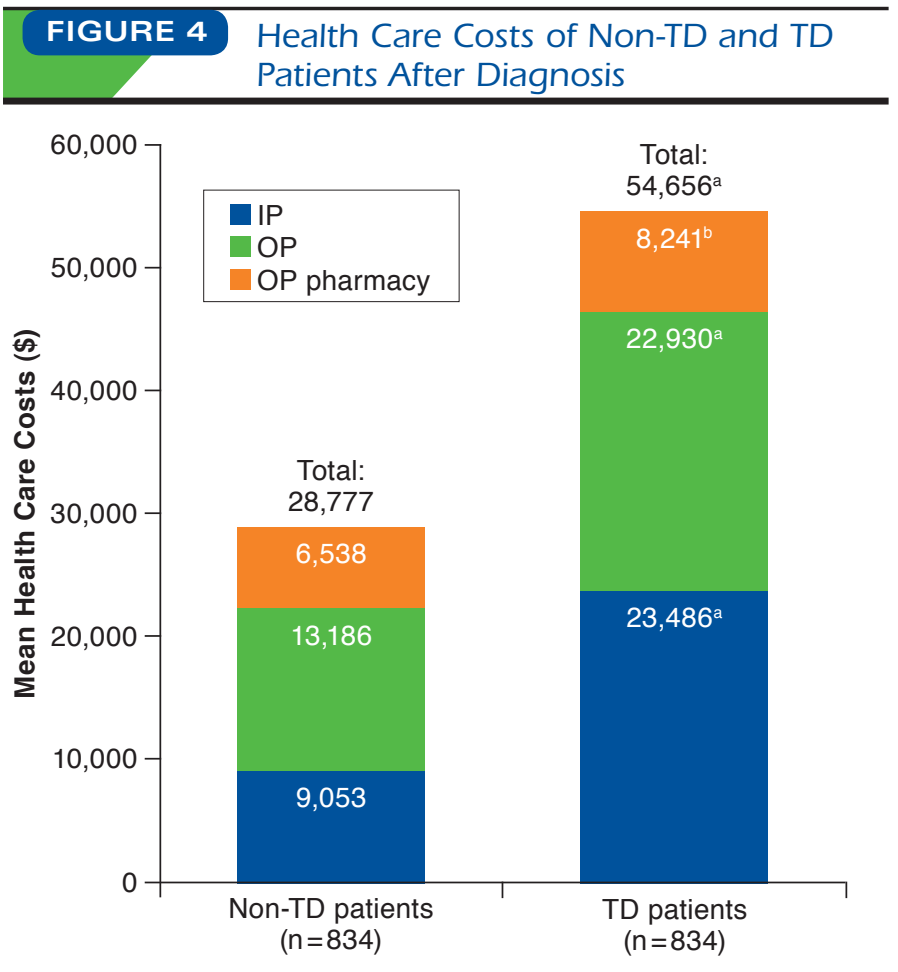

Note: Mean total health care costs subdivided into costs for OP pharmacy; OP and IP admissions per patient of matched TD versus matched non-TD cohorts. Any discrepancy in the total mean health care costs is due to rounding.

aP $<0.001$ compared with non-TD patients.

${ }^{b} \mathrm{P}<0.01$.

$E R=$ emergency room; $I P=$ inpatient; $O P=$ outpatient $; D=$ tardive dyskinesia .

TD-specific treatments (use of tetrabenazine, clonazepam, and amantadine) in the post-index period.

\section{Discussion}

This retrospective claims-based study used real-world data to examine the economic burden of TD. Specifically, in-depth and longitudinal information about health care practice across the United States was obtained from 2 large MarketScan Commercial and Medicare Supplemental databases, including data on inpatient and outpatient settings.

Among patients with TD, health care utilization increased significantly in the 12 months after diagnosis compared with the 12 months before diagnosis. This included an overall increase in inpatient admissions, emergency room visits, and outpatient visits.

Consequently, patients with TD also had substantially higher all-cause health care utilization than matched non-TD patients during the post-index period. All types of health care services, such as inpatient admissions, outpatient office visits, and emergency room visits, increased substantially among all patients with TD and per individual after diagnosis compared with non-TD patients. Additionally, the duration of hospitalization per patient was significantly higher for patients diagnosed with TD than for those without TD, and more than $50 \%$ of the outpatient office visits were TD specific.

The rise in health care utilization among patients diagnosed with TD had a notable effect on all-cause health care costs. Driven by expenses for all health care services, such as inpatient and outpatient utilization and outpatient pharmacy, total health care costs were almost twice as high for patients with TD compared with those for patients without TD, despite the low fraction (9.9\%) of TD-specific costs. Future research should examine specific drivers of costs, such as which diagnoses or medication types contribute to the increased costs most significantly.

The expenses for TD-specific treatments most likely do not reflect the actual TD-related costs because psychotic symptoms are rarely solely related to TD in patients with other underlying mental health conditions. These real-world data suggest that a substantial economic burden is associated with TD diagnosis. TD-related costs are expected to continue to rise, since TD incidence and prevalence are likely to grow concomitantly with the increasing number of patients treated with antipsychotic drugs, which may be attributed to the rise in off-label (e.g., anxiety) and newer on-label (e.g., bipolar disorder) use. ${ }^{18,19}$ Further research should examine the underlying factors resulting in higher health care resource use and costs in patients diagnosed with TD. It is crucial that patients receive efficacious and safe treatments for TD to ensure cost-effectiveness. The novel VMAT2 inhibitors valbenazine and deutetrabenazine are generally well tolerated and effective in reducing TD-related involuntary movements. ${ }^{22,23}$ However, high costs may limit accessibility; more analyses will be needed to assess how patients benefit from the new treatment options. ${ }^{13}$

\section{Limitations}

This study has limitations common to any retrospective analysis. Only those individuals with commercial health coverage or private Medicare supplemental coverage were included. Consequently, results of this analysis may not be generalizable to patients with other forms of insurance or without health insurance coverage. As with any claims databases, the MarketScan research databases rely on administrative claims data for clinical detail, which are subject to data coding limitations and data entry error.

\section{Conclusions}

Patients identified as being diagnosed with TD have significantly higher health care utilization and costs in the 12 months after diagnosis than in the 12 months before diagnosis. Their rates of health care utilization and costs are also higher than those of patients without TD. 


\section{Authors}

BENJAMIN CARROLL, PharmD, Teva Pharmaceuticals, Frazer, Pennsylvania, and DEBRA E. IRWIN, PhD, MSPH, Truven Health Analytics, an IBM Watson Health Company, Durham, North Carolina.

AUTHOR CORRESPONDENCE: Benjamin Carroll, PharmD, Teva Pharmaceuticals, 41 Moores Rd., Malvern, PA 19355. Tel.: 610.727.6329; E-mail: Benjamin.Carroll02@tevapharm.com.

\section{DISCLOSURES}

This study was funded by Teva Pharmaceuticals (Petach Tikva, Israel). Truven Health Analytics, an IBM Watson Health Company, received payment from Teva Pharmaceuticals for the analysis in this study. Carroll is employed by Teva Pharmaceuticals and Irwin is employed by Truven Health Analytics, an IBM Watson Health Company.

\section{ACKNOWLEDGMENTS}

The authors thank Dana Meyen, PhD (Chameleon Communications International, with funding from Teva Pharmaceuticals) for editorial assistance in the preparation of this report.

\section{REFERENCES}

1. Waln O, Jankovic J. An update on tardive dyskinesia: from phenomenology to treatment. Tremor Other Hyperkinet Mov (N Y). 2013;3.

2. Lerner PP, Miodownik C, Lerner V. Tardive dyskinesia (syndrome): current concept and modern approaches to its management. Psychiatry Clin Neurosci. 2015;69(6):321-34.

3. Aquino CC, Lang AE. Tardive dyskinesia syndromes: current concepts. Parkinsonism Relat Disord. 2014;20(Suppl 1):S113-17.

4. Zutshi D, Cloud LJ, Factor SA. Tardive syndromes are rarely reversible after discontinuing dopamine receptor blocking agents: experience from a university-based movement disorder clinic. Tremor Other Hyperkinet Mov (N Y). 2014:4:266.

5. Browne S, Roe M, Lane A, et al. Quality of life in schizophrenia: relationship to sociodemographic factors, symptomatology and tardive dyskinesia. Acta Psychiatr Scand. 1996;94(2):118-24.

6. Youssef HA, Waddington JL. Morbidity and mortality in tardive dyskinesia: associations in chronic schizophrenia. Acta Psychiatr Scand. 1987;75(1):74-77.

7. Ballesteros J, Gonzalez-Pinto A, Bulbena A. Tardive dyskinesia associated with higher mortality in psychiatric patients: results of a meta-analysis of seven independent studies. J Clin Psychopharmacol. 2000;20(2):188-94.
8. Caroff SN, Hurford I, Lybrand J, Campbell EC. Movement disorders induced by antipsychotic drugs: implications of the CATIE schizophrenia trial. Neurol Clin. 2011;29(1):127-48

9. Gilbert PL, Harris M, McAdams L, Jeste DV. Neuroleptic withdrawal in schizophrenic patients: a review of the literature. Arch Gen Psychiatry. 1995;52(3):173-88.

10. Schooler NR. Maintenance medication for schizophrenia: strategies for dose reduction. Schizophr Bull. 1991;17(2):311-24.

11. Touma KTB, Scarff JR. Valbenazine and deutetrabenazine for tardive dyskinesia. Innov Clin Neurosci. 2018;15(5-6):13-16.

12. Xenazine (tetrabenazine) tablets, for oral use. Lundbeck. Revised September 2018. Available at: https://www.lundbeck.com/upload/us/files/ pdf/Products/Xenazine_PI_US_EN.pdf. June 4, 2019.

13. Institute for Clinical and Economic Review. Tardive dyskinesia: reportat-a-glance. December 2017. Available at: https://icer-review.org/material/ td-raag/. Accessed May 23, 2019

14. Carbon M, Hsieh CH, Kane JM, Correll CU. Tardive dyskinesia prevalence in the period of second-generation antipsychotic use: a meta-analysis J Clin Psychiatry. 2017;78(3):e264-e278.

15. Tarsy D, Lungu C, Baldessarini RJ. Epidemiology of tardive dyskinesia before and during the era of modern antipsychotic drugs. Hand Clini Neurol. 2011;100:601-16.

16. Saltz BL, Robinson DG, Woerner MG. Recognizing and managing antipsychotic drug treatment side effects in the elderly. Prim Care Companion J Clin Psychiatry. 2004;6(Suppl 2):14-19.

17. Jankelowitz SK. Treatment of neurolept-induced tardive dyskinesia. Neuropsychiatr Dis Treat. 2013;9:1371-80.

18. Alexander GC, Gallagher SA, Mascola A, Moloney RM, Stafford RS. Increasing off-label use of antipsychotic medications in the United States, 1995-2008. Pharmacoepidemiol Drug Safety. 2011;20(2):177-84.

19. Domino ME, Swartz MS. Who are the new users of antipsychotic medications? Psychiatr Serv. 2008;59(5):507-14.

20. Yu AP, Atanasov P, Ben-Hamadi R, Birnbaum H, Stensland MD, Philips G. Resource utilization and costs of schizophrenia patients treated with olanzapine versus quetiapine in a Medicaid population. Value Health. 2009;12(5):708-15.

21. Rosenheck RA, Leslie DL, Sindelar J, et al. Cost-effectiveness of secondgeneration antipsychotics and perphenazine in a randomized trial of treatment for chronic schizophrenia. Am J Psychiatry. 2006;163(12):2080-89.

22. Hauser RA, Factor SA, Marder SR, et al. KINECT 3: a phase 3 randomized, double-blind, placebo-controlled trial of valbenazine for tardive dyskinesia. Am J Psychiatry. 2017;174(5):476-84.

23. Fernandez HH, Factor SA, Hauser RA, et al. Randomized controlled trial of deutetrabenazine for tardive dyskinesia: the ARM-TD study. Neurology. 2017;88(21):2003-10. 
Health Care Resource Utilization and Costs for Patients with Tardive Dyskinesia

APPENDIX ICD-9-CM Codes Used in Medical and Pharmacy Claims

\begin{tabular}{|c|c|c|}
\hline Code & Description & Comorbidity \\
\hline $300.0 x$ & Anxiety & \multirow{2}{*}{ Anxiety } \\
\hline 293.84 & Anxiety disorder & \\
\hline $250.0 x-250.39$ & Diabetes mellitus (mild to moderate) & \multirow{3}{*}{ Diabetes } \\
\hline $250.7 x$ & Diabetes mellitus (mild to moderate) & \\
\hline $250.4 x-250.69$ & Diabetes mellitus with complications & \\
\hline 332.1 & Extrapyramidal symptoms & \multirow{8}{*}{$\begin{array}{l}\text { Extrapyramidal } \\
\text { symptoms }\end{array}$} \\
\hline $333.0-333.3$ & Extrapyramidal symptoms & \\
\hline $333.5-333.7$ & Extrapyramidal symptoms & \\
\hline 333.81 & Extrapyramidal symptoms & \\
\hline 333.82 & Extrapyramidal symptoms & \\
\hline 333.83 & Extrapyramidal symptoms & \\
\hline 333.89 & Extrapyramidal symptoms & \\
\hline 333.9 & Extrapyramidal symptoms & \\
\hline $272.0 x-272.4 x$ & Hyperlipidemia & Hyperlipidemia \\
\hline $401.0 \mathrm{x}$ & Hypertension & \multirow{8}{*}{ Hypertension } \\
\hline $401.1 x$ & Hypertension & \\
\hline $401.9 x$ & Hypertension & \\
\hline $402.00-405.99$ & Hypertension & \\
\hline $437.2 \mathrm{x}$ & Hypertension & \\
\hline $642.00-642.04$ & Hypertension & \\
\hline $642.10-642.24$ & Hypertension & \\
\hline $642.70-642.94$ & Hypertension & \\
\hline $290 . x$ & Dementia & \multirow{7}{*}{ Dementia } \\
\hline $294.1 x-294.2 x$ & Dementia & \\
\hline 310.0 & Dementia & \\
\hline 331.2 & Dementia & \\
\hline 331.9 & Dementia & \\
\hline 780.93 & Dementia & \\
\hline 797 & Dementia & \\
\hline $296.0 x-296.1 x$ & Bipolar disorder & \multirow{6}{*}{ Bipolar } \\
\hline $296.4 x-296.7 x$ & Bipolar disorder & \\
\hline $296.80-296.81$ & Bipolar disorder & \\
\hline$\underline{296.89}$ & Bipolar disorder & \\
\hline $296.9 x$ & Bipolar disorder & \\
\hline 301.13 & Bipolar disorder & \\
\hline $296.2 x$ & Major depressive disorder single episode & \multirow{2}{*}{$\begin{array}{l}\text { Major depressive } \\
\text { disorder }\end{array}$} \\
\hline $296.3 x$ & Major depressive disorder recurrent episode & \\
\hline 294.9 & Unspecified persistent mental disorders due to conditions classified elsewhere & \multirow{11}{*}{ Cognitive disorder } \\
\hline 331.83 & Mild cognitive impairment, so stated & \\
\hline 310.1 & Personality change due to conditions classified elsewhere & \\
\hline 299.00 & Autistic disorder, current or active state & \\
\hline 299.01 & Autistic disorder, residual state & \\
\hline 299.10 & Childhood disintegrative disorder, current or active state (includes Heller's syndrome) & \\
\hline 299.11 & Childhood disintegrative disorder, residual state & \\
\hline 299.80 & $\begin{array}{l}\text { Other specified pervasive developmental disorders, current or active state (includes } \\
\text { Asperger's syndrome) }\end{array}$ & \\
\hline 299.81 & Other specified pervasive developmental disorders, residual state & \\
\hline 299.90 & Unspecified pervasive developmental disorder, current or active state & \\
\hline 299.91 & Unspecified pervasive developmental disorder, residual state & \\
\hline 295.xx & Schizophrenia & Schizophrenia \\
\hline $\begin{array}{l}300.1 x-300.9,301.0-301.12,301.2 x-301.9 \\
\text { 302.xx, 305.1x-305.9x, 306.xx, } \\
\text { 307.xx-309.xx, 310.2-310.9, 311.xx-316.xx }\end{array}$ & Other neurotic disorders & $\begin{array}{l}\text { Other neurotic } \\
\text { disorder }\end{array}$ \\
\hline $\begin{array}{l}\text { 292.xx, 293.0x-293.83, 293.89-293.9, } \\
\text { 294.0x, 294.8 }\end{array}$ & Other organic psychotic disorders (drug-induced/transient mental disorders) & $\begin{array}{l}\text { Other organic } \\
\text { psychotic disorder }\end{array}$ \\
\hline 296.82, 297.x-298.x & Other psychoses & Other psychoses \\
\hline 290-319 & Mental disorders & Mental disorder \\
\hline
\end{tabular}

816a Journal of Managed Care \& Specialty Pharmacy JMCP July 2019 Vol. 25, No. 7 www.jmcp.org 